\title{
An Oxygenated and Transportable Machine Perfusion System Fully Rescues Liver Grafts Exposed to Lethal Ischemic Damage in a Pig Model of DCD Liver Transplantation
}

\author{
Philippe Compagnon, MD,PhD, ${ }^{1,2} *$ Eric Levesque, MD, ${ }^{2,3}$ Hassen Hentati, MD, ${ }^{1,2}$ \\ Mara Disabato, MD, ${ }^{1}$ Julien Calderaro, MD,PhD ${ }^{4}$ Cyrille Feray, MD,PhD, ${ }^{5}$ Anne Corlu, PhD, ${ }^{6}$ \\ José Laurent Cohen, $\mathrm{PhD},{ }^{2}$ Ismail Ben Mosbah, $\mathrm{PhD},{ }^{2,7 \dagger}$ and Daniel Azoulay, MD,PhD, ${ }^{1,2 \dagger}$
}

${ }^{1}$ Service de Chirurgie Digestive, Hépato-Bilio-Pancréatique et Transplantation Hépatique, Hôpital Henri Mondor, Assistance Publique-Hôpitaux de Paris, Université Paris-Est, Créteil, France

${ }^{2}$ Institut Mondor Recherche Biomédicale (IMRB), INSERM U955, Université Paris-Est, Créteil, France

${ }^{3}$ Service de Réanimation Digestive, Hôpital Henri Mondor, Assistance Publique-Hôpitaux de Paris, Université Paris-Est, Créteil, France

${ }^{4}$ Service d'Anatomopathologie, Hôpital Henri Mondor, Assistance Publique-Hôpitaux de Paris, Université Paris-Est, Créteil, France

${ }^{5}$ Service d'Hépatologie, Hôpital Henri Mondor, Assistance Publique-Hôpitaux de Paris, Université Paris-Est, Créteil, France

${ }^{6}$ Inserm, UMR991, Foie, Métabolismes et Cancer, Hôpital Pontchaillou, Université de Rennes 1, Rennes, France

${ }^{7}$ Biopredic International, Rennes, France 
*Corresponding Author:

Pr Philippe COMPAGNON, M.D., Ph.D.

Service de Chirurgie Digestive et Hépato-Biliaire - Transplantation Hépatique

Hôpital Henri Mondor - Assistance Publique-Hôpitaux de Paris, Université Paris-Est

51 Avenue du Maréchal de Lattre de Tassigny, 94010 Créteil - France

Tel: + 331498142 05, Fax: + 33149812432

E-mail: philippe.compagnon@hmn.aphp.fr

$\uparrow$ These authors contributed equally to this work.

\section{AUTHORSHIP:}

\section{Author's specific contributions:}

PC and IBM designed the study, performed the experiments, analyzed the data, interpreted the data and wrote the manuscript. EL performed the experiments, analyzed the data and participated in drafting the manuscript. $\mathrm{HH}, \mathrm{MD}, \mathrm{JC}$ and $\mathrm{AC}$ performed the experiments and interpreted the data. CF and JC analyzed the data, participated in drafting the manuscript and revised it critically. DA contributed to the conception of the study, interpreted the data and revised the article critically.

\section{Disclosures:}

The authors have no conflicts of interest to declare.

\section{Funding:}

- Eusapharma (Limonest, France) partly funded the experimental work.

- Bridge to Life "Europe" (Ltd, London, United Kingdom) graciously provided the preservation solution for machine perfusion. 


\section{ABBREVIATIONS:}

ALT, alanine aminotransferase

AST, aspartate aminotransferase

ATP, adenosine triphosphate

CHOP, C/EBP homologous protein

DCD, donor after circulatory death

DNA, deoxyribonucleic acid

EIP, end-ischemic perfusion

ELISA, enzyme-linked immunosorbent assay

ER, endoplasmic reticulum

GLDH, glutamate dehydrogenase

GRP78, 78-kDa glucose-regulated/binding immunoglobulin protein

H\&E, hematoxylin/eosin

HMP, hypothermic machine perfusion

HOPE, hypothermic oxygenated perfusion

HTK, histidine-tryptophan-ketoglutarate

INR, international normalized ratio

LT, liver transplantation

MDA, malondialdehyde

MP, machine perfusion

NMP, normothermic machine perfusion

PiCCO, pulse index continuous cardiac output

PNF, primary nonfunction

PNP, purine nucleotide phosphorylase

ROS, reactive oxygen species 
SCS, simple cold storage

SEC, sinusoidal endothelial cells

SOD, superoxide dismutase

TBARS, thiobarbituric acid reactive substances

TNF- $\alpha$, tumor necrosis factor alpha

TUNEL, immunohistological terminal deoxynucleotidyl transferase-mediated dutp-biotin nick end labeling

WI, warm ischemia 


\section{ABSTRACT}

Background: Control of warm ischemia (WI) lesions that occur with donation after circulatory death (DCD) would significantly increase the donor pool for liver transplantation. We aimed to determine whether a novel, oxygenated and hypothermic machine perfusion device (HMP Airdrive system) improves the quality of livers derived from DCDs using a large animal model.

Methods: Cardiac arrest was induced in female large white pigs by IV injection of potassium chloride. After 60min of WI, livers were flushed in situ with HTK and subsequently preserved either by simple cold storage (WI-SCS group) or HMP (WI-HMP group) using Belzer-MPS ${ }^{\mathrm{TM}}$ solution. Liver grafts procured from heart-beating donors and preserved by SCS served as controls. After $4 \mathrm{hr}$ of preservation, all livers were transplanted.

Results: All recipients in WI-SCS group died within 6 hours after transplantation. In contrast, the HMP device fully protected the liver against lethal ischemia/reperfusion injury, allowing $100 \%$ survival rate. A postreperfusion syndrome was observed in all animals of the WI-SCS group but none of the control or WI-HMP groups. After reperfusion, HMP-preserved livers functioned better, and showed less hepatocellular and endothelial cell injury, in agreement with better-preserved liver histology relative to WI-SCS group. In addition to improved energy metabolism, this protective effect was associated with an attenuation of inflammatory response, oxidative load, endoplasmic reticulum stress, mitochondrial damage and apoptosis.

Conclusions: This study demonstrates for the 1st time the efficacy of the HMP Airdrive system to protect liver grafts from lethal ischemic damage prior to transplantation in a clinically relevant DCD model. 


\section{INTRODUCTION}

Liver grafts procured after circulatory death (DCDs) might increase the pool of organs by up to $20 \% .{ }^{1}$ However, many centers remain reluctant to use those grafts due to jeopardized graft function posttransplantation. ${ }^{2}$ By definition, warm ischemia (WI) is unavoidable for such organs with an increased risk of primary nonfunction and ischemic cholangiopathy, leading to increased morbidity and ultimately graft failure. ${ }^{3,4}$ To prevent ischemia-related complications, hypothermic machine perfusion (HMP) has regained interest to overcome the limits of standard simple cold storage (SCS). Unlike SCS, HMP maintains oxygen supply to the cold organ, preserving its ability to synthesize ATP. ${ }^{5}$ HMP has been shown to be more effective than SCS in experimental models, ${ }^{6-10}$ and preliminary clinical studies ${ }^{11-14}$ Guarrera et al, ${ }^{11}$ were the 1 st to report in humans the safety of an HMP device without active oxygenation of the perfusion fluid. Another device using an oxygenated perfusate delivered at low pressure through the portal vein (system HOPE) has been used in the clinical setting and demonstrated better protection of DCD livers than SCS. ${ }^{13,14}$ Notably, these 2 devices are not transportable and can be used only at the receiving hospital after SCS, prior to implantation. Intuitively, perfusion of the liver beginning at the donor site and maintained until the implantation might be more protective than end-ischemic perfusion (EIP). To this end, an innovative HMP device (Airdrive - Amsterdam, The Netherlands) has been developed as a mobile and disposable perfusion system for solid organ storage (Fig. 1A). This device has shown a beneficial effect in experimental hypothermic preservation of kidney and liver allografts. ${ }^{15,16}$ Further, the Airdrive is the 1st transportable oxygenated HMP unit for liver preservation. Using a clinically relevant DCD model in the pig, we determined whether this new perfusion system improves donor liver viability for transplantation. 


\section{MATERIALS AND METHODS}

\section{Animals}

This study was performed in female large white pigs $(25-30 \mathrm{~kg})$ in accordance with French Ministry of Agriculture and local ethical committee (protocol number 14-032). Animals were fasted with free access to water 24 hours prior to surgery. All animals were premedicated with intramuscular 20mg ketamine. General anesthesia was induced with intravenous administration of 4-5 mg/kg propofol. After endotracheal intubation and mechanical ventilation, anesthesia was maintained by inhalation of isoflurane $(0.2-1.5 \%)$ and repeated bolus of sufentanil depending on the animals' need.

\section{Experimental design}

Donor death was induced by intravenous injection of potassium chloride under full anesthesia. Cardiac arrest occurred immediately and mechanical ventilation was stopped. The animal was subsequently left untouched for 45 minutes, resulting in a time interval of 60 minutes between cardiac arrest and in situ cooling. No heparin was given to animals prior or during the warm ischemic insult. Liver allografts procured from heart beating donors and preserved by SCS served as controls. Animals were divided into 3 groups:

i. In control group $(n=6)$, livers from heart beating donors were cold flushed with HTK solution (Eusapharma, Limonest, France) immediately after aortic cross clamping (ie, without warm ischemia), and then preserved by SCS in HTK solution at $4{ }^{\circ} \mathrm{C}$.

ii. In WI-SCS group (n=4), livers were exposed to 60 minutes WI, cold flushed with HTK solution, and subsequently preserved by SCS in HTK solution at $4{ }^{\circ} \mathrm{C}$.

iii. In WI-HMP group ( $\mathrm{n}=6$ ), livers were exposed to 60 minutes WI, cold flushed with HTK solution, and subsequently preserved by HMP in combination with University of 
Wisconsin machine perfusion solution (Belzer-MPS ${ }^{\mathrm{TM}}$, Bridge To Life, Columbia, SC, USA) under hypothermic conditions $\left(2-6^{\circ} \mathrm{C}\right)$ (Fig. 1A).

After 4 hours of preservation, all liver allografts were orthotopically transplanted into recipient animals.

\section{Donor operation}

Harvesting procedure and bench preparation were performed in all animals as previously described. ${ }^{17}$ In MP livers, cannulation of the hepatic artery and portal vein was accomplished with single use and specifically designed perfusion cannulas of appropriate size.

Simple cold storage (SCS)

After washout, grafts were placed in a sterile bag filled with $500 \mathrm{~mL}$ of HTK solution, and subsequently stored at $4^{\circ} \mathrm{C}$.

\section{Machine perfusion with the Airdrive}

The machine perfusion device Airdrive (Amsterdam Medical Center spin-off SME Oxiplenish) was used (Fig. 1A). This lightweight transportable (fully operational $11 \mathrm{Kg}$ ) and disposable MP system is based on an oxygen-driven positive displacement pump that allows pressure-controlled pulsatile perfusion and oxygenation of the perfusion medium by means of a specially designed oxygenator. ${ }^{15,16}$ The oxygen is provided by a standard onboard 2-L pressurized cylinder containing medical grade oxygen (Air Liquide). A 12-V, nonrechargeable battery powers the gas valves and pressure feedback systems via a proportional-integralderivative controller. Cold packs are used to keep the temperature of the system at less than $10^{\circ} \mathrm{C}$. The allograft is perfused until implantation via portal and artery systems using 1.5 liter of refrigerated Belzer-MPS ${ }^{\mathrm{TM}}$. The vascular resistance generated by the liver graft regulates the 
perfusion flow, not exceeding 200ml/min. A software-embedded maximum pressure limit prevents overshoot of pressure to the organ. Perfusion flow and pressure, intrahepatic vascular resistance, perfusion time as well as temperature are continuously recorded and visualized on a LCD display throughout HMP (Fig. 1A). Samples of the perfusate can be collected at regular time interval and analyzed for oxygenation using an automatic analyzer.

\section{Orthotopic liver transplantation (LT)}

All animals underwent the same orthotopic transplantation procedure including flush with cold albumin before reperfusion, as previously described. ${ }^{17}$ Electrocardiographic activity, body temperature, and diuresis were continuously monitored. In each recipient animal, polyethylene catheters were placed in the left internal jugular vein for infusion and left carotid artery for continuous monitoring. Immediately before removal of the native liver, a passive veno-venous shunt was established between the portal vein and left external jugular vein to maintain stable hemodynamic conditions during the anhepatic phase. As well, $500 \mathrm{~mL}$ of plasmion solution was infused into the recipient just before and during the anhepatic phase A Pulse index Continuous Cardiac Output (PiCCO) system (Pulsion Medical Systems SE, France) was used to monitor heart rate, arterial blood pressure and cardiac output. Sedation was stopped and the animals were extubated immediately after operation, and subsequently taken to their cage with heat lamps. Analgesia was given by means of subcutaneous injection of $0.3 \mathrm{mg}$ buprenorphine $1 \mathrm{~h}$ after tracheal extubation. No immunosuppression was given after LT.

\section{Monitoring of animal and liver allograft function}

Continuous monitoring was performed for 5 days after transplantation. Arterial blood gas, glycemia and electrolyte analysis was performed at regular interval during the 1st hours posttransplant. The primary outcome measure was 5-day survival. Animals were monitored daily 
with regards to primary nonfunction (PNF) defined as an aggravated form of reperfusion injury resulting in irreversible graft failure: irreversible metabolic acidosis $(\mathrm{pH}<7.1$ and lactate $>7$ $\mathrm{mmol} / \mathrm{L}$ ), irreversible hypoglycemia, minimal or absent bile production and severe coagulopathy (prothrombin time $<20 \%$, INR $>4$ ) with no identifiable technical cause. ${ }^{18}$

Blood samples were collected before laparotomy (baseline) and at 5, 60, 180 minutes after reperfusion, and daily thereafter until postoperative day-5 and/or sacrifice of the animal. Blood was immediately centrifuged, and the plasma was frozen and stored at $-20 \circ \mathrm{C}$ until analysis.

Liver biopsies were collected prior to (baseline) and at the end of the preservation period, and after LT (60 min after reperfusion and at postoperative day-5 in surviving animals). They were either snap frozen in liquid nitrogen and stored at $-80^{\circ} \mathrm{C}$ or preserved in $10 \%$ formaldehyde for further analysis.

Recipients were sacrificed under general anesthesia by an i.v. overdose of sodium pentobarbital at the end of the study period, or earlier when they were judged to be suffering. An autopsy was performed in all cases.

\section{Biochemical determinations}

Hepatocellular injury (AST, ALT), liver (INR, total bilirubin) and kidney function (creatinine) were determined using standard biochemical methods (clinical grade). Sinusoidal endothelial cells (SEC) injury was evaluated by the measurement of Purine Nucleotide Phosphorylase (PNP) activity using an enzymatic method. ${ }^{19}$ To evaluate energy metabolism, we quantified Adenosine Triphosphate (ATP) reserves in livers after preservation as well as ATP 
recovery after transplantation, using a bioluminescence diagnostic kit (Sigma Aldrich, St Quentin, France). Tumor necrosis factor alpha (TNF- $\alpha)$ is a well-known mediator during I/R injury. ${ }^{20}$ TNF- $\alpha$ levels were quantified using porcine Enzyme-Linked Immunosorbent Assay (ELISA) kits from BD Bio-sciences (San Diego, CA, USA). Lipid peroxidation was used as an indirect measurement of the oxidative stress induced by reactive oxygen species (ROS), ${ }^{21}$ and was determined in whole liver homogenates by the quantification of malondialdehyde (MDA) using the Thiobarbituric Acid Reactive Substances (TBARS) assay kit from Interchim (Montluçon, France). Catalase and superoxide dismutase (SOD) activities were determined by using respectively colorimetric and spectrophotometric assay kit from Interchim (Montluçon, France). We looked at Endoplasmic reticulum (ER) stress through the determination of the ER chaperone $78-\mathrm{kDa}$ glucose-regulated/binding immunoglobulin protein (GRP78) and C/EBP homologous protein (CHOP), an effector of ER stress involved in apoptosis and deoxyribonucleic acid (DNA) damage, ${ }^{22}$ using porcine ELISA kits from MyBioSource (San Diego, CA, USA). A fluorometric assay kit was obtained from Biovision (Palo Alto, CA, USA) to determine liver caspase-12 activity, this protease being involved in the initiation of ER stressinduced cell death. ${ }^{23}$ Glutamate dehydrogenase (GLDH), an enzyme that indicates mitochondrial damages, was measured in plasma samples using an enzymatic method as previously described. ${ }^{24}$ Cytochrome $\mathrm{C}$ activity in plasma was evaluated using porcine ELISA kit from MyBioSource (San Diego, CA, USA). Frozen tissue samples weighing $100 \mathrm{mg}$ were homogenized in $1 \mathrm{~mL}$ of Phosphate-buffered saline, and supernatant was collected to determine caspase- 9 activity by using a colorimetric assay kit from Biovision (Palo Alto, CA, USA). Liver caspase-3 activity was measured using a colorimetric assay kit (Biovision, Palo Alto, CA, USA). 
Histology and TUNEL assay

Histological hematoxylin/eosin (H\&E) and immunohistological terminal deoxynucleotidyl Transferase-mediated dUTP-biotin Nick End Labeling (TUNEL) analysis were performed on liver tissue specimens to appraise the degree of hepatic injury by optical microscopy and apoptosis, respectively. Histological lesions on stained sections were graded on a scale as previously described, taking into account the extent and severity of 7 morphologic features and resulting in a score ranging between 0 and $47 .{ }^{25,26}$ Apoptosis was assessed by TUNEL assay (Roche Molecular Biochemical's, Mannheim, Germany) as described elsewhere. ${ }^{21}$ Histological evaluation of damage score and ratio of the apoptotic cell number were performed in a blinded fashion by 2 experienced liver pathologists.

\section{Statistical analysis}

Data were expressed as the means \pm SD. Data were analyzed using Graph Pad Prism software (Version 6.0; Graph Pad, San Diego, CA). Statistical analyses were performed by a Kruskal-Wallis 1-way analysis-of-variance-by-ranks-test followed by a nonparametric MannWhitney-Wilcoxon U-test when the overall comparison of groups was significant. A survival analysis was calculated according to a Kaplan Meier (Log-rank test). A p value below 0.05 was considered significant.

\section{RESULTS}

\section{Operative characteristics}

The operative characteristics did not significantly differ between groups (Table 1). 


\section{Perfusion Parameters}

The perfusion parameters in the Airdrive system were stable during the perfusion period (Fig. 1, B and C). Blood gas analysis before cannulation of the liver yielded a pO2 of $342 \pm 22$ $\mathrm{mmHg}$. After graft placement in the Airdrive, the pO2 dropped immediately to $126 \pm 15 \mathrm{mmHg}$ and remained stable thereafter.

\section{Airdrive improved hemodynamic profiles after transplantation}

During the anhepatic phase, hemodynamic and respiratory parameters were similar between groups. A postreperfusion syndrome was observed in all animals that received a SC stored liver after 60min WI (WI-SCS group) compared to none of the control or HMP groups. At 1-hour postreperfusion, mean arterial pressure and cardiac output decreased in WI-SCS group compared to controls ( $49 \pm 15$ vs. $67.6 \pm 24 \mathrm{mmHg}, \mathrm{p}=0.04$ and $2.62 \pm 1.5$ vs. $4.21 \pm 0.58 \mathrm{l} / \mathrm{min}$, $\mathrm{p}=0.02$, respectively) and machine perfused livers $(49 \pm 15$ vs. $59 \pm 13 \mathrm{mmHg}, \mathrm{p}=0.33$ and 2.62 \pm 1.5 vs. $4.03 \pm 1.35 \mathrm{l} / \mathrm{min}, \mathrm{p}=0.5$, respectively). Accordingly, catecholamine needs after reperfusion were increased in WI-SCS group $(34.0 \pm 17.7 \mathrm{mg})$ relative to controls $(14.75 \pm 9.88$ $\mathrm{mg}, \mathrm{p}<0.05)$ and WI-HMP group $(12.75 \pm 4.5 \mathrm{mg}, \mathrm{p}<0.05)$.

\section{Airdrive improved liver graft recovery and animal survival after transplantation}

All the animals in the WI-SCS group experienced PNF, and died within 6hr after transplantation. No deaths were due to procedure-related causes. In contrast and like controls, all animals from the WI-HMP group showed full functional recovery and survived until sacrifice at 5 days. 
Airdrive improved liver graft function and attenuated liver cell damage after transplantation

After reperfusion, MP livers functioned better than the WI-SCS livers, as reflected by INR profiles and serum bilirubin levels (Fig. 2, A and B). Hepatic dysfunction observed in WISCS group (Fig. 2, A and B) was associated with acute renal failure (Fig. 2C). Recipients from WI-SCS group also developed more severe hepatocellular injury. The increase in transaminases was continuous until death and the difference with controls was highly significant (Fig. 2, D and E). In contrast, Airdrive caused a marked reduction in enzyme release, the postoperative course of ALT and AST being comparable between MP livers and controls (Fig. 2, D and E). MP livers also exhibited less endothelial cell injury relative to the WI-SCS group, as evidenced by serum PNP levels (Fig. 2F).

\section{Airdrive better preserved liver histology}

Histological changes following preservation and reperfusion were in line with hepatocellular and endothelial injury (Fig. 3 A and B). Histology in WI-SCS group revealed marked vascular congestion, sinusoidal dilatation and parenchymal cell loss, predominating in centrilobular areas (Fig. 3, A and B) and more pronounced after 1hr reperfusion (Fig. 3B). The damage score was significantly higher in WI-SCS group relative to controls, either at the end of preservation $(22.66 \pm 0.74$ vs. $7.41 \pm 0.37 ; \mathrm{p}<0.05)$ (Fig. 3C) or after reperfusion $(39.58 \pm 1.12$ vs. $15 \pm 0.77 ; \mathrm{p}<0.05$ ) (Fig. $3 \mathrm{D}$ ). A better maintenance of liver integrity was observed in MP livers, compared to WI-SCS group (Fig. 3, A and B). At 1h postreperfusion, we could observe well-preserved hepatocytes cords with slight sinusoidal dilatation and moderate vascular congestion in WI-HMP group (Fig. 3B). The damage score in MP livers was significantly lower relative to SC stored livers ( $p<0.05)$, both at the end of the preservation period $(24 \pm 2$ vs. $8 \pm 1$; p $<0.05$ ) (Fig. 3C) and after 60min reperfusion (39.58 \pm 5 vs. $22.33 \pm 0.54$; p<0.05) (Fig. 3D). At 5 days posttransplantation, a microscopic examination of the MP livers showed complete 
resolution of histological abnormalities, and the MP livers were similar to controls (Figure S1 A and C, SDC, http://links.lww.com/TP/B430).

\section{Airdrive better maintained energy metabolism}

The HMP device attenuated depletion of hepatic ATP content during cold preservation, and improved ATP recovery after reperfusion compared to WI-SCS group ( $p<0.05$, Fig. 4A).

\section{Airdrive attenuated inflammatory response}

An attenuation of inflammatory response was observed in WI-HMP group, as indicated by a significant reduction in cytokine TNF- $\alpha$ levels, both in liver tissue (Fig. 4B) and plasma (Fig. 4C).

\section{Airdrive attenuated oxidative load and ER stress}

Tissue MDA content was significantly reduced in WI-HMP group, both at the end of preservation and after $60 \mathrm{~min}$ reperfusion, thereby translating lower oxidative stress $(\mathrm{p}<0.05$ vs. WI-SCS, Fig. 5A). MP livers also exhibited higher catalase and SOD activities compared to WISCS group (Fig. 5, B and C).

HMP also lowered expression of ER stress markers GRP78 and CHOP at 60min postreperfusion (Fig. 5, D and E). Accordingly, caspase-12 activity was significantly reduced in WI-HMP group at 60 min postreperfusion ( $\mathrm{p}<0.05$ vs. WI-SCS, Fig. 5F).

\section{Airdrive better preserved mitochondrial integrity and protected against hepatic apoptosis}

MP livers exhibited lower GLDH activity, cytochrome C and caspase-9 cleavage release both at the end of preservation and after 60 min reperfusion ( $<<0.05$ vs. WI-SCS, Fig.6, A, B and 
C), expressing a better preservation of mitochondrial integrity. Accordingly, the lower levels of cleaved caspase-9 in HMP group were associated with decreased caspase- 3 activity ( $p<0.05$, Fig. 6D). Consequently, Airdrive attenuated hepatic apoptosis as indicated by a significant reduction in the percentage of TUNEL-positive hepatocytes both at the end of preservation (9.4\% vs. $18.5 \%$ in WI-SCS group, p $<0.05$ ) (Fig. 7, A and C), and after 60min reperfusion (7.5\% vs. $53.1 \%$ in WI-SCS group, $\mathrm{p}<0.05$ ) (Fig. 7, B and D). At 5 days posttransplantation, the percentage of TUNEL-positive cells was similar between the WI-HMP group and controls (Figure S1 B and D, SDC, http://links.lww.com/TP/B430).

\section{DISCUSSION}

This study demonstrates that oxygenated HMP started immediately after procurement fully rescues the pig liver exposed to lethal ischemic damage in a model of DCD liver transplantation, allowing 100\% survival rates, along with improved graft function, decreased hepatocellular and endothelial injury, and better-preserved liver histology. In addition to the improved energy metabolism, the protective effect of early perfusion is associated with attenuated inflammatory response, tissue oxidative load, ER stress, mitochondrial damage and apoptosis.

Several groups are investigating liver MP techniques with different approaches regarding route of perfusion (single vs. dual vessel perfusion), oxygenation, perfusate solutions and temperature. .,6,11,13,27 $^{-1}$

So far, human application of MP remains limited, but promising early experiences have been described using HMP on allografts procured from either brain dead donors ${ }^{11}$ or extended criteria donors including DCDs. ${ }^{12-14}$ There has been parallel enthusiasm for normothermic MP (NMP). ${ }^{28}$ However, it remains unclear whether claimed benefits (the only clinical marker was peak AST) 
are worth the cost, the complexity and the known risks of NMP (ie, potential for bacterial growth, no backup strategy available in case of mechanical failure). In contrast, HMP offers a protective effect of liver grafts by a simple and practical perfusion technique.

The optimal timing and duration of HMP application remain highly debated. With previously mentioned devices, ${ }^{11,13}$ HMP is started after initial SCS for organ transportation. This approach is termed end-ischemic perfusion (EIP) and has the advantage to be performed in the secured environment of the transplant center with all the support needed. Although EIP is logistically attractive, marked injury may have occurred already to the liver and the biliary tree. ${ }^{29}$ We are currently conducting a study using the Airdrive system to compare these 2 strategies, ie, early vs. delay perfusion.

Because it is a lightweight and transportable device, Airdrive permits from a logistical standpoint continuous preservation perfusion from the harvesting location without additional SCS and consequently, allows the prevention or early treatment of graft damage. Animals transplanted with a WI-SCS graft developed severe liver dysfunction and injury as evidenced by histology and transaminases levels, leading to irreversible metabolic acidosis, systemic shock and finally death of all recipients within few hours. In contrast, the use of HMP throughout the preservation period fully prevented these events.

The Airdrive system is simple and reliable since it is based on an oxygen-driven positive displacement pump that allows autoregulation of pressure and flow profiles. Pressure-controlled perfusion in hypothermic machine liver perfusion is a critical point to minimize the risk of shear stress, mediator release and SEC damage. ${ }^{9,30}$ SEC are also particularly vulnerable to hypothermia which induces serious morphological alterations leading to impaired graft microcirculation, Kupffer cell activation, and adhesion molecules up-regulation. ${ }^{31}$ All these disorders contribute to neutrophil infiltration and finally hepatocyte death upon reperfusion. ${ }^{32}$ PNP activity was 
significantly lower in recipients of the WI-HMP group, indicating better preservation of SEC. Serum PNP levels have been shown to be inversely related to SEC viability. ${ }^{32}$ Although the exact mechanism remains unclear, an indirect protective effect mediated through improved microcirculation is plausible. It may also reflect a better maintenance of the antioxidant system of livers subjected to I/R injury. ${ }^{32}$ Since polyunsaturated fatty acids in the cell membrane are vulnerable to lipid peroxidation, oxidative stress leads to liver cell death very rapidly. ${ }^{21,33}$ This free radical-mediated process was significantly lowered in MP livers, an attenuation that was partly explained by higher catalase and SOD activities. An increase in the activities of both enzymes has been shown to attenuate I/R liver injury. ${ }^{34}$ Airdrive also attenuated inflammatory response. Similar findings have been reported by Guarrera et al. ${ }^{35}$

In addition to pulsatile dual perfusion, our HMP device provides continuous oxygenation of the perfusion medium. Oxygen supply under hypothermic conditions is a key strategy to decrease reperfusion injury, particularly in organs exposed to WI before procurement, such as DCD livers. ${ }^{10,36}$ The optimal amount of oxygen needed remains unclear. Our system yielded a pO2 of about $340 \mathrm{mmHg}$ (ie, 45kPA) before cannulation of the liver, a level that corresponds to reported levels by others, ranging between 10 and $100 \mathrm{kPa} .{ }^{37} \mathrm{HMP}$ is 1 way to maintain oxygen supply to the cold organ and therefore its capacity to synthesize ATP, an indispensable energy source to the cell for the maintenance of its structural and functional integrity. ${ }^{38}$ ATP is also recognized as an independent risk factor for initial graft dysfunction. ${ }^{39}$ In accordance with previous reports, ${ }^{40-42}$ Airdrive improved energy metabolism during cold preservation as well as after reperfusion. This portable HMP device also facilitated ER adaptation. ER stress is an adaptive response to the accumulation of misfolded proteins within the ER. Increasing evidences suggest its implication in the pathogenesis of hepatic I/R injury associated with $\mathrm{LT}^{43,44}$ 
To further understand the mechanisms leading to liver cell protection, we evaluated mitochondrial damage and apoptotic cell death. Apoptosis is a hallmark of I/R-induced graft damage. $^{45,46}$ Release of cytochrome $\mathrm{c}$ from mitochondria is an early event of the apoptotic process in response to oxidative stress. ${ }^{47,48}$ It further induces caspase- 9 activation that in turn leads to the activation of the effector caspase- 3 , a downstream event in the apoptotic cascade. ${ }^{49}$ Airdrive attenuated hepatic apoptosis as evidenced by the significant reduction in the percentage of TUNEL-positive hepatocytes as well as decrease of cleaved caspase-3 levels compared to WIHMP group. The lower GLDH activity, cytochrome c release and caspase-9 cleavage observed in MP livers suggests that mitochondria might also be an important target through which Airdrive exerts its cytoprotective effect. The team of PA Clavien has previously reported mitochondrial protection with HMP. ${ }^{30}$

Being disposable, Airdrive minimizes potential infectious hazard. Besides, this device is cost-saving compared to available HMP systems since it does not require important preuse investments, full-time specialized supervision, maintenance, return transport and cleaning.

The design of our study was limited to the analysis of early graft injury and 5-day survival, omitting information on later biliary injury, as this complication typically occurs several weeks after LT. Whether our device also prevents late biliary injury in the setting of DCDs needs further studies.

In conclusion, we show for the 1st time the efficacy of the HMP Airdrive device to rescue, otherwise lethal, ischemic injured DCD livers in a clinically relevant transplantation model. HMP with Airdrive with cannulation of the liver graft in the donor operating room may 
help to achieve the full potential of HMP. This novel portable system should also improve results of transplantation from other type of extended criteria donors and allow safe extension of what was previously considered the limits of safe cold preservation for liver allografts.

\section{ACKNOWLEDGMENTS:}

We are grateful to Drs. Mara Costa and Mariana Cornet for their assistance in the operating room. We also thank Philippe Druelle and Philippe Mario for taking good care of animals. 


\section{REFERENCES}

1. Monbaliu D, Pirenne J, Talbot D. Liver transplantation using donation after cardiac death donors. J Hepatol. 2012; 56(2):474-485.

2. Goldberg DS, Abt PL. Improving outcomes in DCDD liver transplantation: there can only be strength in numbers. Am J Transplant. 2014; 14(5):1016-1020.

3. de Vera ME, Lopez-Solis R, Dvorchik I, et al. Liver transplantation using donation after cardiac death donors: long-term follow-up from a single center. Am J Transplant. 2009; 9(4):773-781.

4. Jay CL, Lyuksemburg V, Ladner DP, et al. Ischemic cholangiopathy after controlled donation after cardiac death liver transplantation: a meta-analysis. Ann Surg. 2011; 253(2):259-264.

5. Schlegel A, Kron P, Dutkowski P. Hypothermic machine perfusion in liver transplantation. Curr Opin Organ Transplant. 2016; 21(3):308-314.

6. Compagnon P, Clément B, Campion JP, Boudjema K. Effects of hypothermic machine perfusion on rat liver function depending on the route of perfusion. Transplantation. 2001; 72(4):606-614.

7. Bessems M, Doorschodt BM, van Vliet AK, van Gulik TM. Improved rat liver preservation by hypothermic continuous machine perfusion using polysol, a new, enriched preservation solution. Liver Transpl. 2005; 11(5):539-546.

8. de Rougemont O, Breitenstein S, Leskosek B, et al. One hour hypothermic oxygenated perfusion (HOPE) protects nonviable liver allografts donated after cardiac death. Ann Surg. 2009; 250(5):674-683.

9. Fondevila C, Hessheimer AJ, Maathuis MH, et al. Hypothermic oxygenated machine perfusion in porcine donation after circulatory determination of death liver transplant. Transplantation. 2012; 94(1):22-29. 
10. Schlegel A, Graf R, Clavien PA, Dutkowski P. Hypothermic oxygenated perfusion (HOPE) protects from biliary injury in a rodent model of DCD liver transplantation. J Hepatol. 2013; 59(5):984-991.

11. Guarrera JV, Henry SD, Samstein B, et al. Hypothermic machine preservation in human liver transplantation: the first clinical series. Am J Transplant. 2010; 10(2):372-381.

12. Guarrera JV, Henry SD, Samstein B, et al. Hypothermic machine preservation facilitates successful transplantation of "orphan" extended criteria donor livers. Am J Transplant. 2015; 15(1):161-169.

13. Dutkowski P, Schlegel A, de Oliveira M, Müllhaupt B, Neff F, Clavien PA. HOPE for human liver grafts obtained from donors after cardiac death. J Hepatol. 2014; 60(4):765-772.

14. Dutkowski P, Polak WG, Muiesan P, et al. First comparison of hypothermic oxygenated PErfusion versus static cold storage of human donation after cardiac death liver transplants: an international-matched case analysis. Ann Surg. 2015; 262(5):764-770.

15. Schreinemachers MC, Doorschodt BM, Florquin S, et al. Pulsatile perfusion preservation of warm ischaemia-damaged experimental kidney grafts. Br J Surg. 2010; 97(3):349-358.

16. Dirkes MC, Post IC, Heger M, van Gulik TM. A novel oxygenated machine perfusion system for preservation of the liver. Artif Organs. 2013; 37(8):719-724.

17. Audet M, Alexandre E, Mustun A, et al. Comparative evaluation of Celsior solution versus Viaspan in a pig liver transplantation model. Transplantation. 2001; 71(12):1731-1735.

18. Monbaliu D, Libbrecht L, De Vos R, et al. The extent of vacuolation in non-heart-beating porcine donor liver grafts prior to transplantation predicts their viability. Liver Transpl. 2008; 14 (9):1256-1265.

19. Nakagami M, Morimoto T, Mitsuyoshi A, Mashima S, Shimabukuro T, Yamaoka Y. Difference in onset of warm ischemia and reperfusion injury between parenchymal and 
endothelial cells of the liver. Evaluation by purine nucleoside phosphorylase and hyaluronic acid. J Surg Res. 1996; 62(1):118-124.

20. Perry BC, Soltys D, Toledo AH, Toledo-Pereyra LH. Tumor necrosis factor- $\alpha$ in liver ischemia/reperfusion injury. J Invest Surg. 2011; 24(4):178-188.

21. Ben Mosbah I, Mouchel Y, Pajaud J, et al. Pretreatment with mangafodipir improves liver graft tolerance to ischemia/reperfusion injury in rat. PLoS One. 2012; 7(11):e50235.

22. Ben Mosbah I, Duval H, Mbatchi SF, et al. Intermittent selective clamping improves rat liver regeneration by attenuating oxidative and endoplasmic reticulum stress. Cell Death Dis. 2014; 5:e1107.

23. Szegezdi E, Fitzgerald U, Samali A. Caspase-12 and ER-stress-mediated apoptosis: the story so far. Ann N Y Acad Sci. 2003; 1010:186-194. Review.

24. Ben Mosbah I, Massip-Salcedo M, Fernández-Monteiro I, et al. Addition of adenosine monophosphate-activated protein kinase activators to University of Wisconsin solution: a way of protecting rat steatotic livers. Liver Transpl. 2007; 13(3):410-425.

25. Imber CJ, St Peter SD, Lopez de Cenarruzabeitia I, et al. Advantages of normothermic perfusion over cold storage in liver preservation. Transplantation. 2002; 73(5):701-709.

26. Liu Q, Vekemans K, Iania L, et al. Assessing warm ischemic injury of pig livers at hypothermic machine perfusion. J Surg Res. 2014; 186(1):379-389.

27. Hoyer DP, Mathe Z, Gallinat A, et al. Controlled oxygenated rewarming of cold stored livers prior to transplantation: first clinical application of a new concept. Transplantation. 2016; 100(1):147-52

28. Ravikumar R, Jassem W, Mergental H, et al. Liver transplantation after ex vivo normothermic machine preservation: a phase 1 (first-in-man) clinical trial. Am J Transplant. 2016; 16(6):1779-1787. 
29. Weeder PD, van Rijn R, Porte RJ. Machine perfusion in liver transplantation as a tool to prevent non-anastomotic biliary strictures: rationale, current evidence and future directions. $J$ Hepatol. 2015; 63(1):265-275.

30. Schlegel A, Dutkowski P. Role of hypothermic machine perfusion in liver transplantation. Transpl Int. 2015; 28(6):677-689.

31. Clavien PA. Sinusoidal endothelial cell injury during hepatic preservation and reperfusion. Hepatology. 1998; 28(2):281-285.

32. Ikeda A, Ueki S, Nakao A, et al. Liver graft exposure to carbon monoxide during cold storage protects sinusoidal endothelial cells and ameliorates reperfusion injury in rats. Liver Transpl. 2009; 15(11):1458-1468.

33. Lee SM, Park MJ, Cho TS, Clemens MG. Hepatic injury and lipid peroxidation during ischemia and reperfusion. Shock. 2000; 13(4):279-284.

34. Yabe Y, Nishikawa M, Tamada A, Takakura Y, Hashida M. Targeted delivery and improved therapeutic potential of catalase by chemical modification: combination with superoxide dismutase derivatives. J Pharmacol Exp Ther. 1999; 289(2):1176-1184.

35. Guarrera JV, Henry SD, Chen SW, et al. Hypothermic machine preservation attenuates ischemia/reperfusion markers after liver transplantation: preliminary results. J Surg Res. 2011; 167(2):e365-e373.

36. Lüer B, Koetting M, Efferz P, Minor T. Role of oxygen during hypothermic machine perfusion preservation of the liver. Transpl Int. 2010(9); 23:944-950.

37. Schlegel A, Kron P, Dutkowski P. Hypothermic oxygenated liver perfusion: basic mechanisms and clinical application. Curr Transplant Rep. 2015; 2(1):52-62.

38. Dickson RC, Bronk SF, Gores GJ. Glycine cytoprotection during lethal hepatocellular injury from adenosine triphosphate depletion. Gastroenterology. 1992; 102(6):2098-2107. 
39. Vajdová K, Graf R, Clavien PA. ATP-supplies in the cold-preserved liver: Along-neglected factor of organ viability. Hepatology. 2002; 36(6):1543-1552.

40. Dutkowski P, Odermatt B, Heinrich T, et al. Hypothermic oscillating liver perfusion stimulates ATP synthesis prior to transplantation. J Surg Res. 1998; 80(2):365-372.

41. Stegemann J, Minor T. Energy charge restoration, mitochondrial protection and reversal of preservation induced liver injury by hypothermic oxygenation prior to reperfusion. Cryobiology. 2009; 58(3):331-336.

42. Westerkamp AC, Karimian N, Matton AP, et al. Oxygenated hypothermic machine perfusion after static cold storage improves hepatobiliary function of extended criteria donor livers. Transplantation. 2016; 100(4):825-35

43. Peralta C, Brenner C. Endoplasmic reticulum stress inhibition enhances liver tolerance to ischemia/reperfusion. Curr Med Chem. 2011; 18(13):2016-2024.

44. Zaouali MA, Boncompagni E, Reiter RJ, et al. AMPK involvement in endoplasmic reticulum stress and autophagy modulation after fatty liver graft preservation: a role for melatonin and trimetazidine cocktail. J Pineal Res. 2013; 55(1):65-78.

45. Gao W, Bentley RC, Madden JF, Clavien PA. Apoptosis of sinusoidal endothelial cells is a critical mechanism of preservation injury in rat liver transplantation. Hepatology. 1998; 27(6):1652-1660.

46. Jaeschke H, Gujral JS, Bajt ML. Apoptosis and necrosis in liver disease. Liver Int. 2004; 24(2):85-89.

47. Ben Mosbah I, Alfany-Fernández I, Martel C, et al. Endoplasmic reticulum stress inhibition protects steatotic and non-steatotic livers in partial hepatectomy under ischemia-reperfusion. Cell Death Dis. 2010; 1:e52; doi:10.1038.

48. Liu X, Kim CN, Yang J, Jemmerson R, Wang X. Induction of apoptotic program in cell-free extracts: requirement for dATP and cytochrome c. Cell. 1996; 86(1):147-157. 
49. Enari M, Sakahira H, Yokoyama H, Okawa K, Iwamatsu A, Nagata S. A caspase-activated DNase that degrades DNA during apoptosis, and its inhibitor ICAD. Nature. 1998; 391(6662):43-50. 
FIGURE 1. Illustration of the HMP Airdrive system (A) and Perfusion parameters (B,C).

FIGURE 2. Liver graft function, hepatocellular and endothelial damage. Airdrive improved liver graft function when compared to WI-SCS group (A,B). The severe hepatic dysfunction observed in WI-SCS group was associated with acute renal failure (C). Machine-perfused livers also exhibited less hepatocellular (D,E) and endothelial cell injury (F) compared to WI-SCS group. The data are presented as the mean \pm SD from individual experiments. $* \mathrm{p}<0.05$ versus controls, $+\mathrm{p}<0.05$ versus WI-SCS.

FIGURE 3. Representative H\&E stained sections (magnification x 200) and damage score of liver grafts. Histological examination revealed marked vascular congestion, sinusoidal dilatation and parenchymal cell loss in WI-SCS group (A,B). These lesions predominated in centrilobular areas (A-B) and were more pronounced at $1 \mathrm{~h}$ posttransplantation (B). The damage score was higher in WI-SCS group relative to controls, either at the end of preservation (C) or after reperfusion (D). Liver histology was better maintained in machine-perfused [MP] livers, compared to WI-SCS group (A-B). The damage score in MP livers was lower relative to WISCS group, both at the end of the preservation period (C) and at $1 \mathrm{~h}$ posttransplantation (D). ${ }^{*} \mathrm{p}<0.05$ versus controls, $+\mathrm{p}<0.05$ versus WI-SCS.

FIGURE 4. Energy metabolism and inflammatory response. Airdrive attenuated depletion of hepatic ATP content during cold preservation and improved ATP recovery after reperfusion compared to WI-SCS group $(\mathrm{p}<0.05)(\mathrm{A})$. The protective effect of the Airdrive was associated with an attenuation of the inflammatory response as indicated by the reduction in cytokine TNF$\alpha$ levels both in liver tissue (B) and plasma (C). The data are presented as the mean \pm SD from individual experiments. $* \mathrm{p}<0.05$ versus controls, $+\mathrm{p}<0.05$ versus WI-SCS. 
FIGURE 5. Oxidative load and Endoplasmic Reticulum (ER) stress. Tissue MDA content was reduced in machine-perfused [MP] livers both at the end of preservation and at $1 \mathrm{~h}$ posttransplantation (A). This attenuation was associated with higher catalase and superoxide dismutase activities in MP livers relative to WI-SCS group (B,C). Airdrive lowered expression of ER stress markers - 78-kDa glucose-regulated/binding immunoglobulin protein (GRP78) and C/EBP homologous protein (CHOP) - at 1h posttransplantation (D-E), and reduced caspase-12 activity at $1 \mathrm{~h}$ postreperfusion $(\mathrm{F})$. The data are presented as the mean $\pm \mathrm{SD}$ from individual experiments. ${ }^{*} \mathrm{p}<0.05$ versus controls, $+\mathrm{p}<0.05$ versus WI-SCS.

FIGURE 6. Mitochondrial integrity. MP livers exhibited lower GLDH activity, cytochrome c release and caspase-9 cleavage (A-C). The lower levels of cleaved caspase-9 in turn attenuated the activation of the effector caspase-3 (D). The data are presented as the mean \pm SD from individual experiments. ${ }^{*} \mathrm{p}<0.05$ versus controls, $+\mathrm{p}<0.05$ versus WI-SCS.

FIGURE 7. Hepatic apoptosis. An increased number of apoptotic liver cells was observed in the WI-SCS group compared to controls at the end of preservation (A) and particularly at $1 \mathrm{~h}$ posttransplantation (B). Conversely, Airdrive significantly reduced the percentage of TUNELpositive hepatocytes both at the end of preservation $(A, C)$, and after $1 \mathrm{~h}$ reperfusion $(B, D)$. The data are presented as the mean \pm SD from individual experiments. ${ }^{*} \mathrm{p}<0.05$ versus controls, + $\mathrm{p}<0.05$ versus WI-SCS. 
(A)

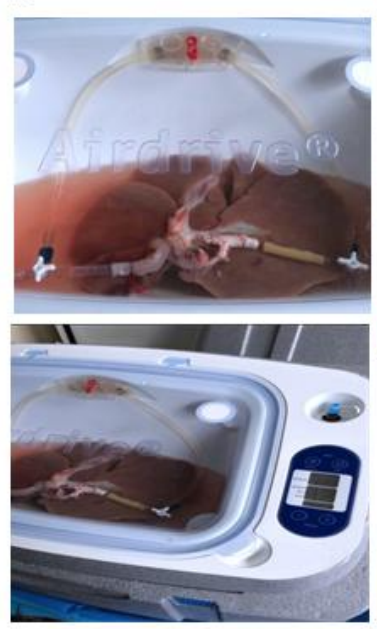

(B)

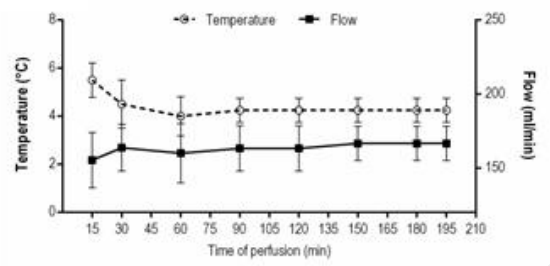

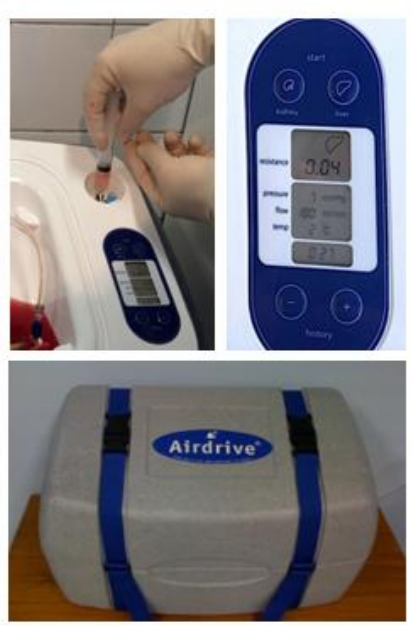

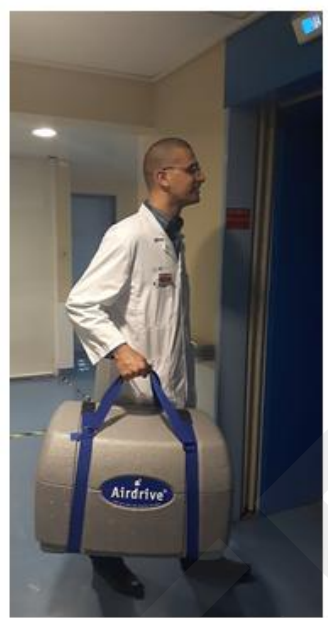

(C)

Figure 1 
(A)

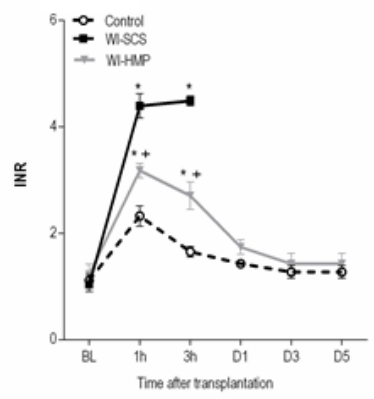

(D)

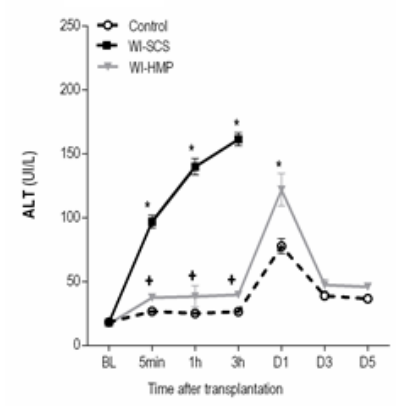

(B)

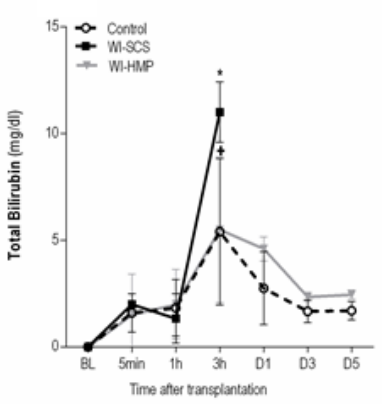

(E)

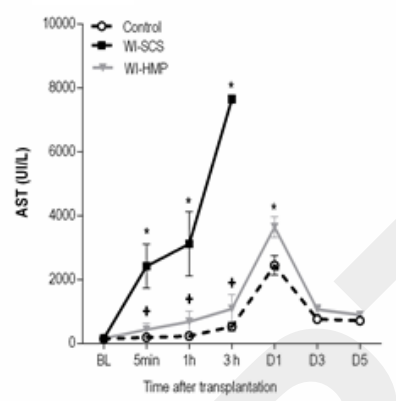

(C)

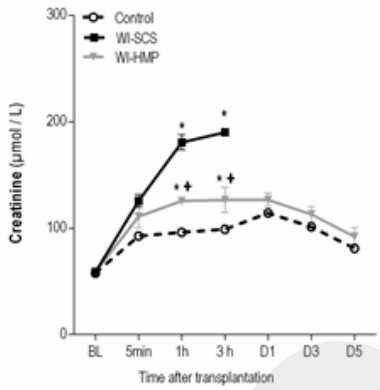

(F)

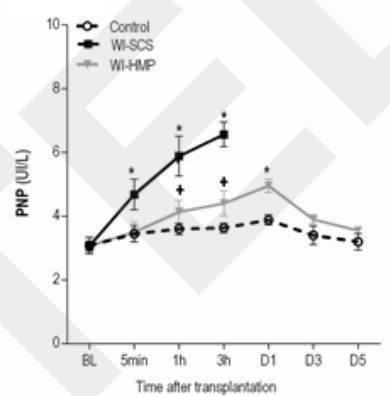

Figure 2 
(A)

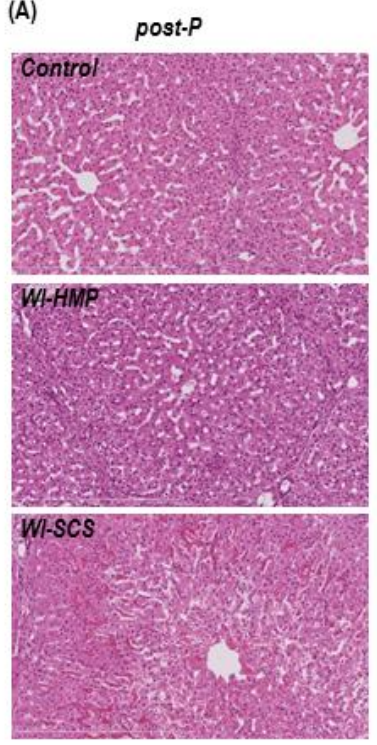

(C)

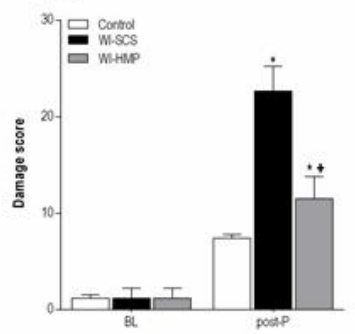

(B) 1 post-Tx
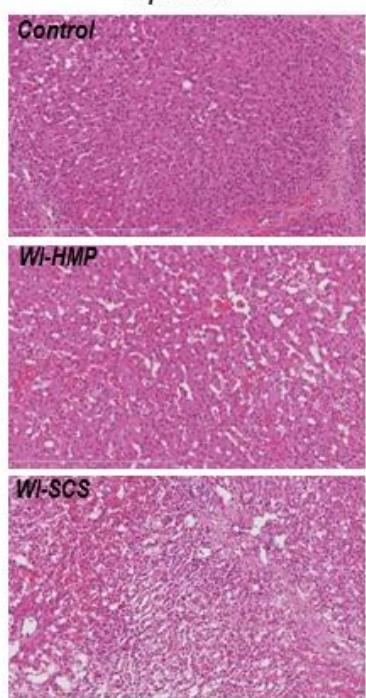

(D)

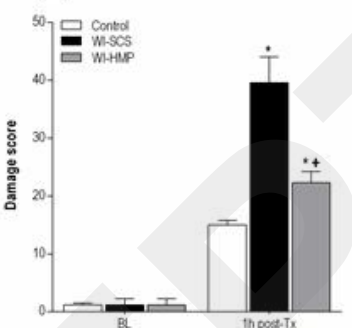

Figure 3 
(A)

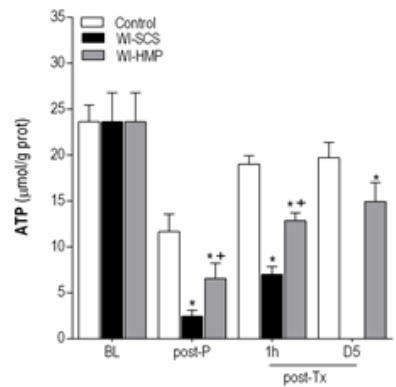

(B)

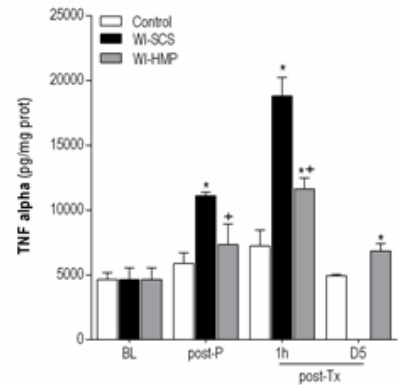

(C)

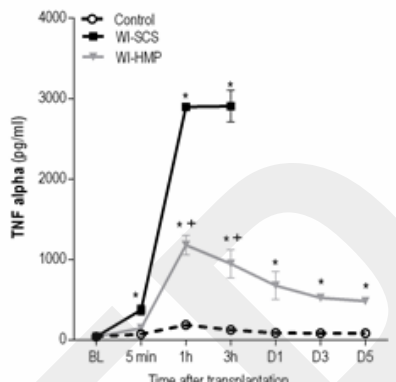

Figure 4 
(A)

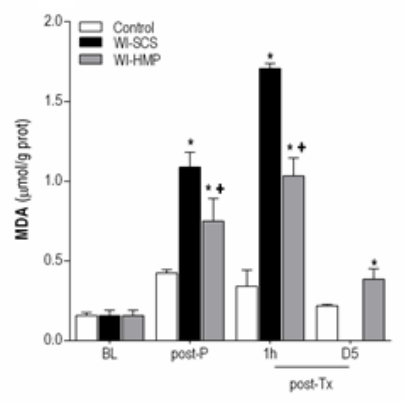

(D)

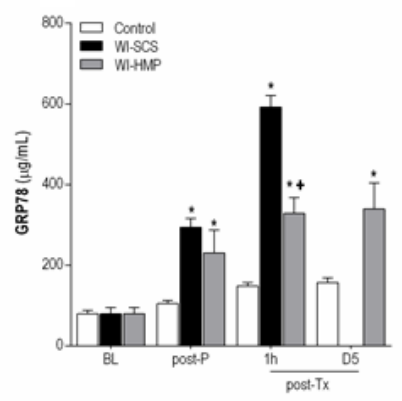

(B)

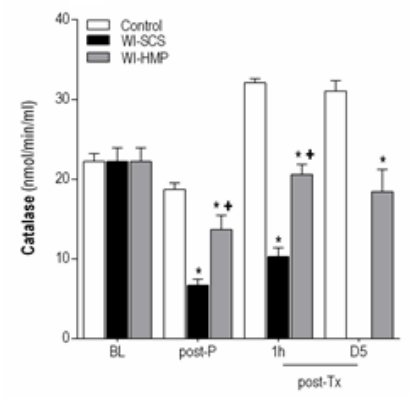

(E)

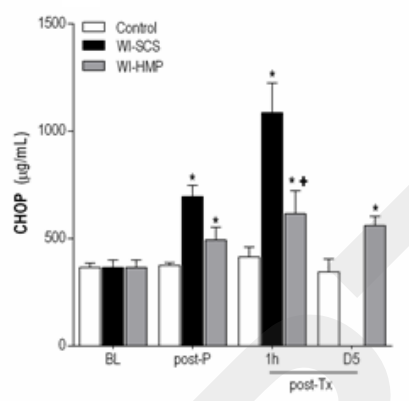

(C)

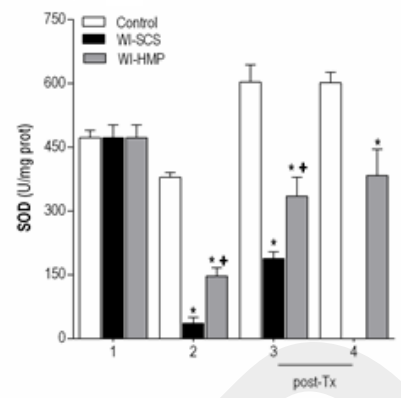

(F)

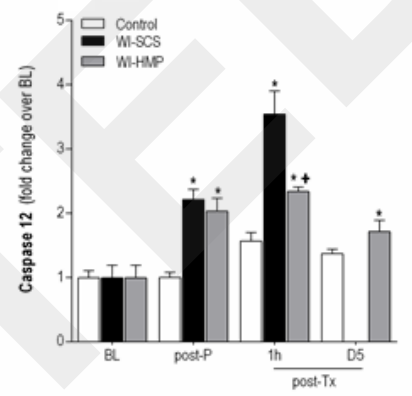

Figure 5 
(A)

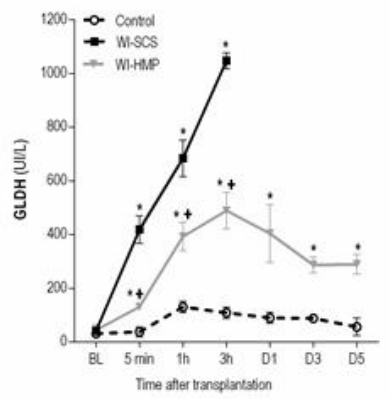

(C)

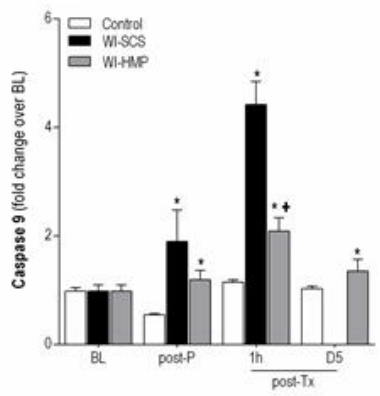

(B)

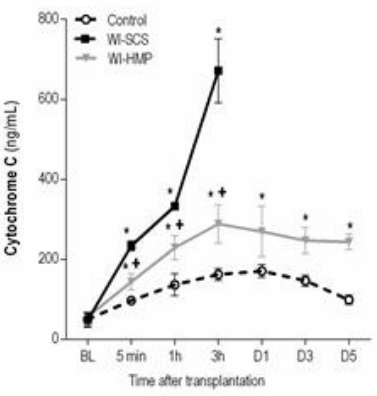

(D)

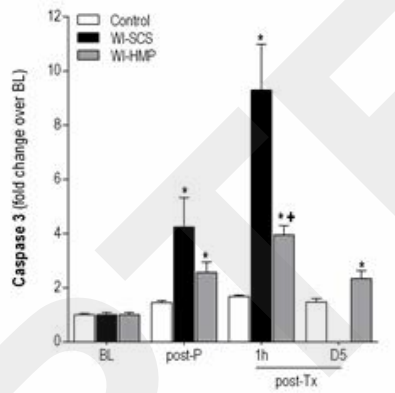

Figure 6 
(A)

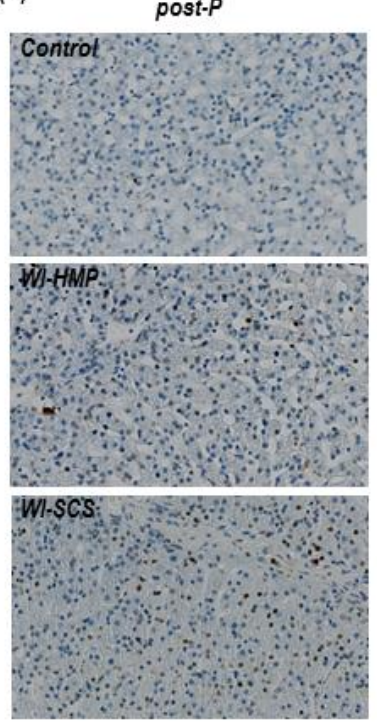

(C)

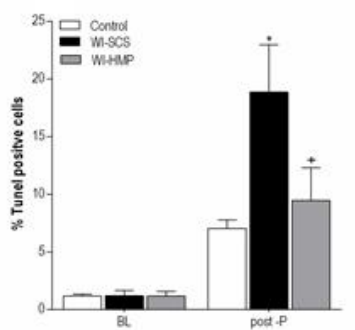

(B)

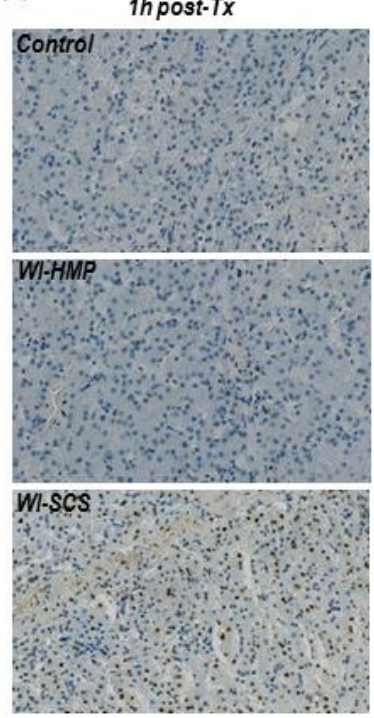

(D)

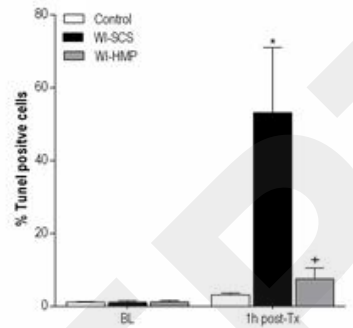

Figure 7 
TABLE 1. Operative characteristics of control, WI-SCS and WI-HMP groups

\begin{tabular}{|c|c|c|c|c|}
\hline & Controls & WI-SCS & WI-HMP & $\mathrm{p}$ \\
\hline Donor Weights (kg) & $33(32-35)$ & $30(27-34)$ & $33(32-34)$ & NS \\
\hline Recipient weights (kg) & $32(31-34)$ & $31(28-34)$ & $32(32-34)$ & NS \\
\hline WIT (min)a & 0 & 60 & 60 & NS \\
\hline CIT $(\min )^{b}$ & $236(230-248)$ & $235(230-245)$ & $35(34-38)$ & NS \\
\hline $\begin{array}{l}\text { Perfusion time with the Airdrive } \\
\text { (min) }\end{array}$ & 0 & 0 & $208(206-211)$ & - \\
\hline Total preservation time $(\min )^{c}$ & $236(230-248)$ & $235(230-245)$ & $243(239-246)$ & NS \\
\hline Length of anhepatic phase $(\min )^{d}$ & $33(31-35)$ & $32(30-35)$ & $32(31-34)$ & NS \\
\hline RWIT (min)e & $28(26-30)$ & $27(24-29)$ & $27(25-30)$ & NS \\
\hline Total operative time (min) & $193(176-205)$ & $192(178-204)$ & $188(175-210)$ & NS \\
\hline
\end{tabular}

a Period between cardiac arrest and cold flush out of the liver in the donor (WI-SCS and WI-HMP groups).

b Period between the chilling of the liver graft in the donor and portal vein unclamping of the liver graft in the recipient (control and WI-SCS groups) or start of machine perfusion (WI-HMP group). In WI-HMP group, CIT corresponded to the time it took to flush, extract, prepare, and place the liver graft on the machine Airdrive.

c Period between the chilling of the liver graft in the donor and portal vein unclamping of the liver graft in the recipient (for all 3 groups). $d$ Period between portal vein clamping of the native liver and portal vein unclamping of the liver graft in the recipient (for all 3 groups).

e Period when the cold liver graft is subjected to body temperature while performing venous vascular reconstruction (until portal vein unclamping). 


\section{SUPPLEMENTARY FIGURE 1:}

After 5-days posttransplantation, microscopic examination showed complete resolution of histological abnormalities $[A, C]$, while TUNEL positive cells were similar between WI-HMP group and controls $[B, D] .{ }^{*}$ $p<0.05$ : versus controls, $+p<0.05$ : versus WI-SCS.

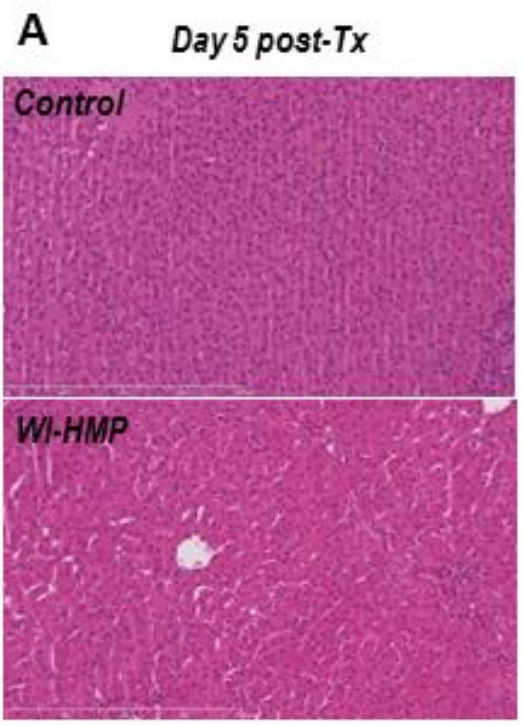

\section{B Day 5 post-Tx}

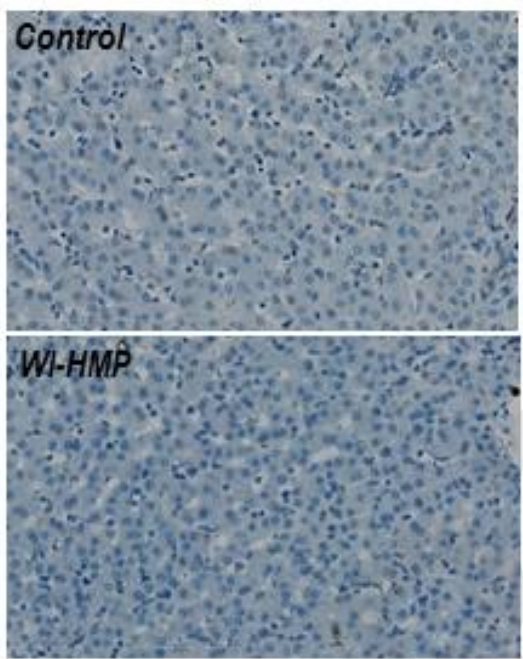

C
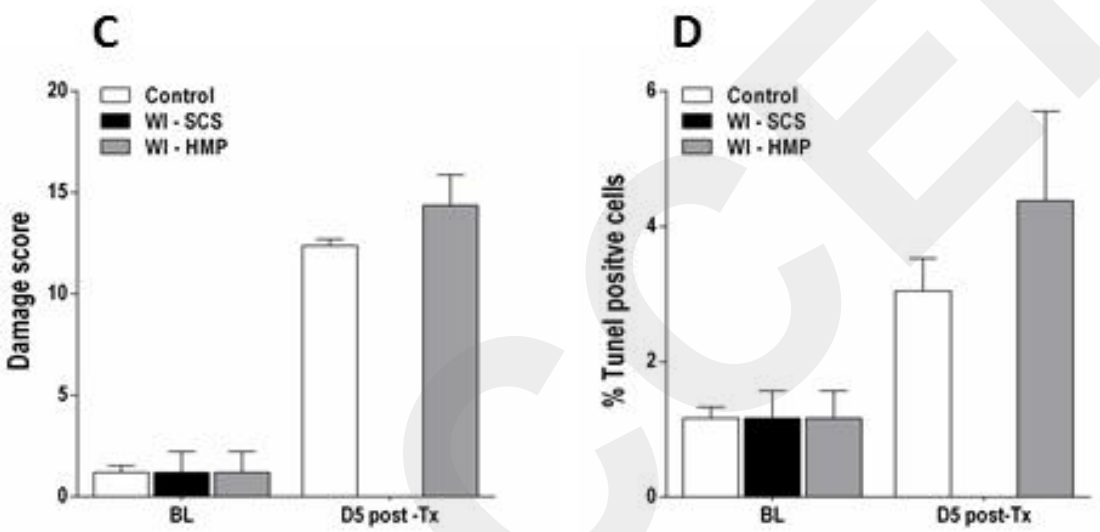

Supplementary Figure 1 